\title{
Inertia-driven spin switching in antiferromagnets
}

\author{
A. V. Kimel ${ }^{1 \star}$, B. A. Ivanov², R. V. Pisarev ${ }^{3}$, P. A. Usachev ${ }^{3}$, A. Kirilyuk ${ }^{1}$ and Th. Rasing ${ }^{1}$
}

It is generally accepted that the fastest way to reorient magnetization is through precessional motion in an external magnetic field ${ }^{1-7}$. In ferromagnets, the application of a magnetic field instantaneously sets spins in motion and, in contrast to the inertial motion of massive bodies, the magnetization can climb over a potential barrier only during the action of a magnetic-field pulse. Here we demonstrate a fundamentally different scenario of spin switching in antiferromagnets, where the exchange interaction between the spins leads to an inertial behaviour. Although the spin orientation hardly changes during the action of an optically generated strong magnetic-field pulse of $100 \mathrm{fs}$ duration, this pulse transfers sufficient momentum to the spin system to overcome the potential barrier and reorient into a new metastable state, long after the action of the stimulus. Such an inertia-based mechanism of spin switching should offer new opportunities for ultrafast recording and processing of magnetically stored information.

The switching of magnetization between two metastable states is very similar to the process of transferring a particle over a potential barrier from one minimum to another (see Fig. 1). The obvious way for such a transfer is to pull the particle over the barrier and let it relax into the new metastable state. Such a scenario is followed in the switching of ferromagnetic spins over $180^{\circ}$ by a magnetic-field pulse ${ }^{2-7}$. For optimal switching speed the pulse width has to be tuned to half the precessional period. Nevertheless, it has been recently shown that if the pulse is shorter than 2 ps the required magnetic field is so strong that the magnetic order collapses and the switching becomes a nondeterministic process ${ }^{3}$.

Alternatively, for massive particles such a potential barrier can be overcome by using their inertia. Even if the interaction between an external stimulus and the particle is so short that the coordinate of the particle hardly changes during the interaction time, as long as the particle acquires sufficient linear momentum and kinetic energy it may overcome the potential barrier afterwards. Were such an inertial mechanism of spin switching feasible, we could control magnetization with magnetic-field pulses much shorter than those required for non-inertial switching.

All magnetically ordered materials can be divided into two groups: ferromagnets and antiferromagnets, the static and dynamic magnetic properties of which are fundamentally different ${ }^{8}$. The dynamics of spins $\mathbf{S}_{i}$ in a ferromagnet is described in terms of the magnetization vector $\mathbf{M}=\Sigma \mathbf{S}_{i}$, the motion of which in an effective magnetic field $\mathbf{H}_{\text {eff }}$ is governed by the Landau-Lifshitz equation $\mathrm{d} \mathbf{M} / \mathrm{d} t=\gamma\left(\mathbf{M} \times \mathbf{H}_{\text {eff }}\right)$ ( $\gamma$ is the gyromagnetic ratio $)^{1,8}$. This equation is of first order with respect to time, $t$, and thus does not contain inertial terms such as acceleration. In contrast, the dynamics of an antiferromagnet with two sublattices $\mathbf{M}_{1}$ and $\mathbf{M}_{2}$ is described in terms of the motion of the antiferromagnetic unit vector $\mathbf{l}=\left(\mathbf{M}_{1}-\mathbf{M}_{2}\right) /\left|\mathbf{M}_{1}-\mathbf{M}_{2}\right|$, which can be written through

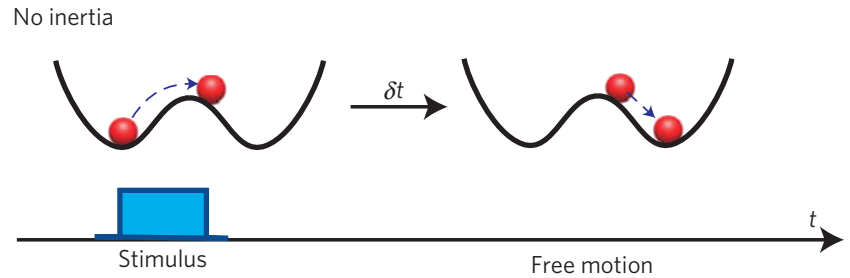

Inertia

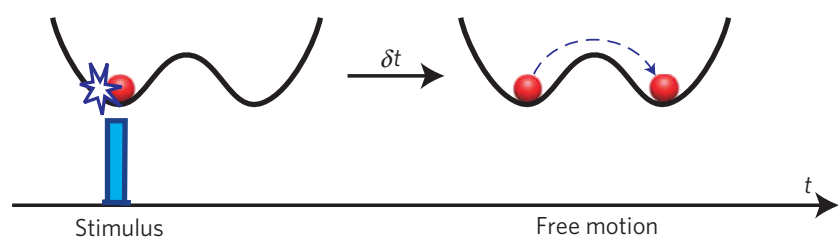

Figure 1 | Non-inertial and inertial models to transport a point mass over a potential. The non-inertial mechanism requires a continuous driving force that pulls the mass over the potential barrier. A similar scenario is realized in magnetization reversal through precessional motion in ferromagnets. In contrast, in the inertial mechanism, during the action of the driving force the coordinate of the particle is hardly changed, but the particle acquires enough momentum to overcome the barrier afterwards.

the variation of the Lagrangian (refs 9, 10 and Supplementary Information) for 1 only,

$$
L=\frac{\hbar}{2 \gamma H_{\mathrm{e}}}\left(\frac{\mathrm{d} \mathbf{l}}{\mathrm{d} t}\right)^{2}+\frac{\hbar}{H_{\mathrm{e}}}\left(\frac{\mathrm{d} \mathbf{l}}{\mathrm{d} t}(\mathbf{l} \times \mathbf{H})\right)-W(\mathbf{l})
$$

whereas the magnetization $\mathbf{M}=\mathbf{M}_{1}+\mathbf{M}_{2}$ is only a slave variable. Here $W(\mathbf{l})$ plays the part of the potential energy, $H_{\mathrm{e}}$ and $\mathbf{H}$ represent the exchange field of the antiferromagnet and the external magnetic field, respectively. This Lagrangian includes the kinetic energy, $\mathrm{dl} / \mathrm{d} t$ (ref. 2), and hence the equation of motion is of second order with respect to time and shows inertial-like motion. This statement is valid even for canted antiferromagnets, where the Dzyaloshinskii-Moriya interaction leads to a non-zero magnetic moment in the ground state. In this case,

$$
W(\mathbf{l})=W_{\mathrm{a}}(\mathbf{l})+\frac{\gamma \hbar}{H_{\mathrm{e}}}\left(\mathbf{H}\left(\mathbf{H}_{\mathrm{D}} \times \mathbf{l}\right)\right)
$$

where $W_{\mathrm{a}}(\mathbf{l})$ describes the effective anisotropy energy and the vector $\mathbf{H}_{\mathrm{D}}$ is the Dzyaloshinskii field of the antiferromagnet, determined by the magnetic symmetry ${ }^{9,10}$. Therefore, antiferromagnetically coupled spins do have inertia and this property

${ }^{1}$ IMM, Radboud University Nijmegen, 6525 ED Nijmegen, The Netherlands, ${ }^{2}$ Institute of Magnetism, NASU, 03142 Kiev, Ukraine, ${ }^{3}$ loffe Physical-Technical

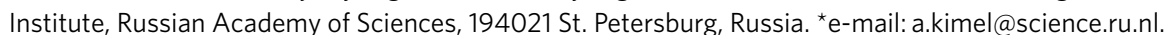



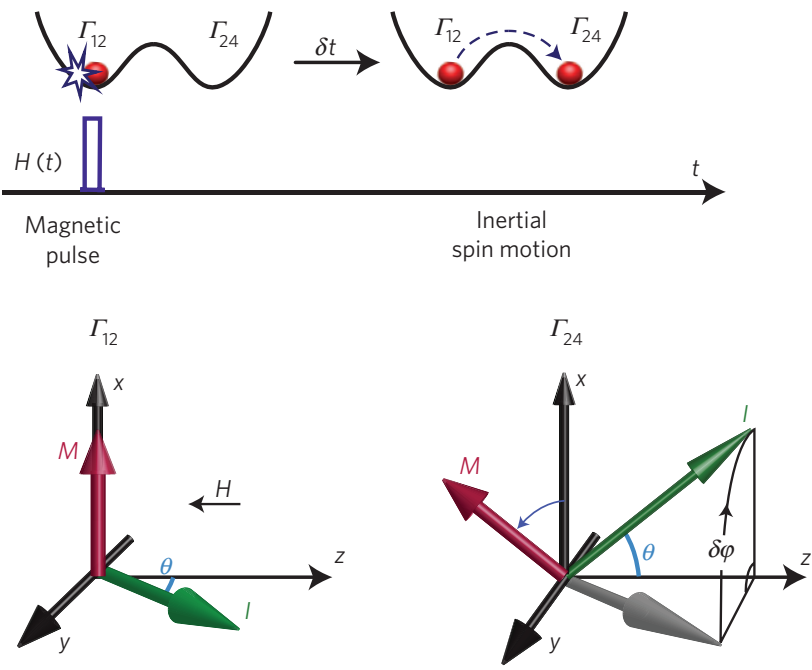

Figure 2 | Spin switching in $\mathbf{H o F e O}_{\mathbf{3}} \cdot \Gamma_{12}$ and $\Gamma_{24}$ are the two metastable phases present between 38 and $52 \mathrm{~K}$. H represents an effective magnetic-field pulse that initiates an inertial motion of spins from the $\Gamma_{12}$ to the $\Gamma_{24}$ magnetic phase. In the $\Gamma_{12}$ phase the antiferromagnetic vector I is in the $y z$ plane and the $z$ component of magnetization $\mathbf{M}$ is zero. A transition towards the $\Gamma_{24}$ phase occurs through a rotation of the $\mathbf{I}$ vector over an angle $\delta \varphi$ towards the new equilibrium in the $z x$ plane with a non-zero $M_{z}$ component.

can be exploited for ultrafast magnetic switching using ultrashort magnetic-field pulses.

Holmium orthoferrite $\mathrm{HoFeO}_{3}$ is an excellent candidate for observing such inertial reorientation of spins. (1) It is a canted antiferromagnet with a Dzyaloshinskii field of about $10 \mathrm{~T}$ parallel to the $y$ axis, leading to a small magnetic moment in the $x z$ plane. Owing to this net magnetization and the last term in equation (2), an external magnetic field can play the part of a driving force for the vector $\mathbf{l}$ and thus trigger an inertial spin motion. (2) Between $T_{1}=38 \mathrm{~K}$ and $T_{2}=58 \mathrm{~K}$ the magnetic system of $\mathrm{HoFeO}_{3}$ is characterized by two thermodynamical potential minima ${ }^{11-13}$. One persists up to $T_{2}=58 \mathrm{~K}$ and corresponds to the $\Gamma_{12}$ magnetic symmetry, with the magnetic moment $\mathbf{M}$ along the $x$ crystallographic axis and the antiferromagnetic vector $\mathbf{l}$ in the $z y$ plane (Fig. 2). In angular variables, $l_{z}=\cos \theta, l_{x}=\sin \theta \sin \varphi$ and $l_{y}=\sin \theta \cos \varphi$, the $\Gamma_{12}$ phase has $\theta=\theta(T) \neq 0, \pi / 2, \ldots$ and $\varphi=0, \pi$ (see Fig. 2). The second potential minimum is present above $T_{1}=38 \mathrm{~K}$ and corresponds to the $\Gamma_{24}$ phase with both $\mathbf{M}$ and $\mathbf{l}$ in the $x z$ plane $\theta=\theta(T) \neq 0, \pi / 2, \ldots$ and $\varphi= \pm \pi / 2$. The transition between these two minima is a first-order phase transition at $T_{\mathrm{t}}=52 \mathrm{~K}$, where $\theta\left(T_{\mathrm{t}}\right)=\theta_{0}$ is about $15^{\circ}-20^{\circ}$ (refs $\left.11-13\right)$, which can be achieved either by heating or by the application of a static magnetic field.

From equations (1) and (2) it can be easily shown that in $\mathrm{HoFeO}_{3}$ between 38 and $58 \mathrm{~K}$ a short magnetic field pulse should trigger an inertial spin-reorientation between the magnetic phases $\Gamma_{12}$ and $\Gamma_{24}$. A laser pulse propagating along the $z$ axis acts as an effective magnetic field $\mathbf{H}=H(t) \mathbf{e}_{z}$ (ref. 16). Supposing $\theta=$ const, it follows from equations (1) and (2) that

$$
\frac{\mathrm{d}^{2} \varphi}{\mathrm{d} t^{2}}+2 \Gamma \frac{\mathrm{d} \varphi}{\mathrm{d} t}+\omega_{0}^{2} \frac{\mathrm{d} w(\varphi)}{\mathrm{d} \varphi}-\frac{\gamma^{2} H_{\mathrm{D}}}{\sin \theta_{0}} H(t) \cos \varphi=0
$$

Here, the acceleration $\mathrm{d}^{2} \varphi / \mathrm{d} t^{2}$ indeed represents the spin inertia, similar to that in the Newton equation of motion for unit mass; the second term presents a viscous force with damping coefficient $\Gamma$; terms with $\mathrm{d} w(\varphi) / \mathrm{d} \varphi$ and $H_{\mathrm{D}}$ represent the restoring and driving force, respectively; $w(\varphi)$ is a dimensionless function proportional to the magnetic anisotropy energy for $\theta=$ const; $\omega_{0}$ is the frequency of the lower antiferromagnetic mode at $T=T_{\mathrm{t}}$. We can see that, for the phase $\Gamma_{12}$ with $\varphi=0$ in the ground state, $H(t)$ acts as a
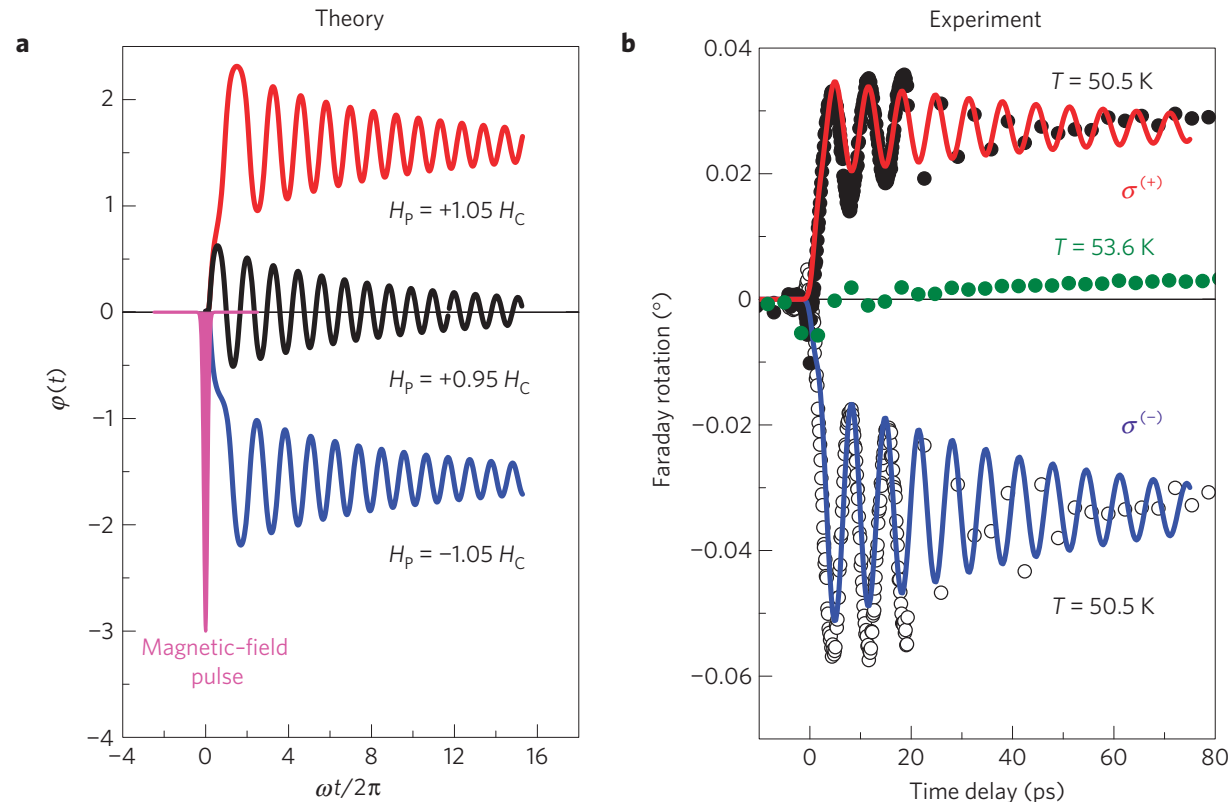

Figure 3 | Theoretical and experimental demonstration of inertia-driven spin switching in $\mathbf{H o F e O}_{\mathbf{3}} \mathbf{.} \mathbf{a}_{\text {, In }}$ the calculations, the reorientation is assumed to be triggered by a 100-fs Gaussian magnetic-field pulse directed along the $z$ axis. Magnetic parameters are taken from refs 11 and 12 . $H_{\mathrm{C}}$ is the critical field required for the transition $\Gamma_{12} \Rightarrow \Gamma_{24}$. The simulations show that for $T=50.5 \mathrm{~K}$ the critical field is equal to $0.6 \mathrm{~T}$. $\mathbf{b}$, In the experiment, the effective magnetic fields along the $z$ axis were generated by right-handed $\sigma^{(+)}$and left-handed $\sigma^{(-)}$circularly polarized laser pulses through the inverse Faraday effect. The trace recorded at $T=53.6 \mathrm{~K}$ for left-handed circularly polarized pump $\sigma^{(-)}$is shown by green points and demonstrates that outside the range between 38 and $52 \mathrm{~K}$ no inertia-driven spin switching is seen. Note that the simulations do not take into account laser-induced heating. The latter is likely the reason for the different oscillation amplitudes observed experimentally for $\sigma^{(-)}$and $\sigma^{(+)}$. 
a

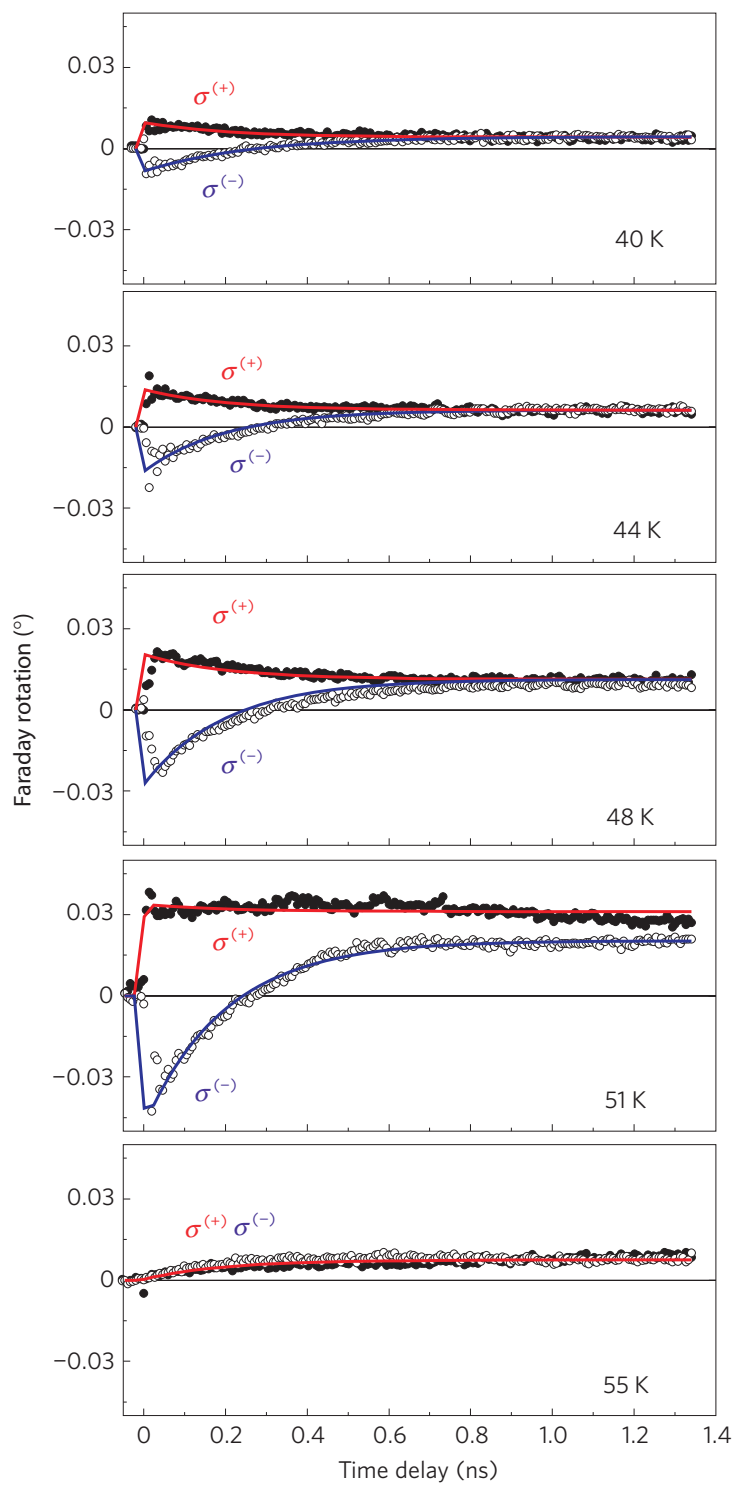

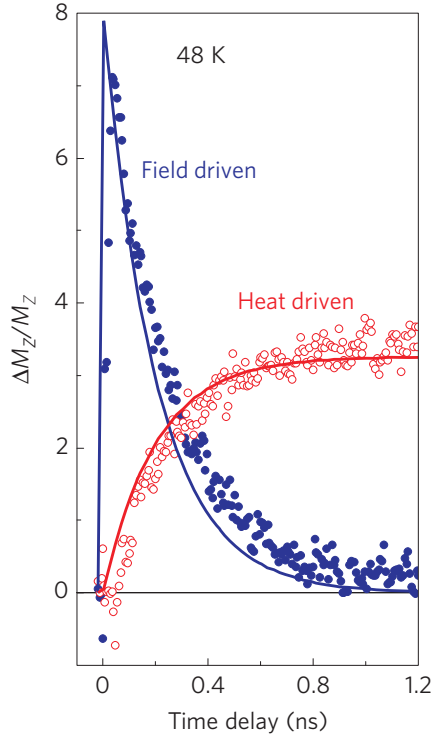

C

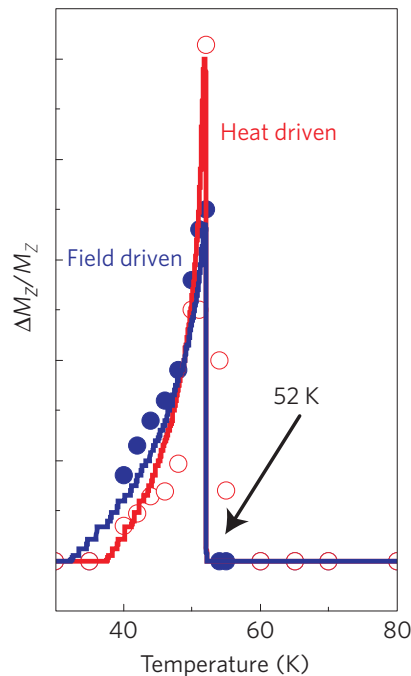

Figure 4 | Laser-induced spin dynamics measured by the Faraday rotation at different temperatures. a, Dynamics of the Faraday rotation triggered by right-handed $\sigma^{(+)}$and left-handed $\sigma^{(-)}$circularly polarized laser pulses. b. Dynamics of spin reorientation driven by laser heating (open circles) and laser-generated magnetic fields (solid circles). c, Temperature dependencies of the spin reorientation driven by laser heating (solid circles) and laser-generated magnetic fields (open circles).

force exciting the dynamics of the 'coordinate' $\varphi$, whereas the effect of the field is negligible for the $\Gamma_{24}$ phase with $\varphi=\pi / 2$ in the ground state. Results of calculations on the basis of equation (3) are shown in Fig. 3a. It is seen that the excitation of the spins by a $100 \mathrm{fs}$ magnetic-field pulse with an amplitude $H_{\mathrm{P}}$ triggers damped spin oscillations and may change the equilibrium value of the angle $\varphi$. The new equilibrium orientation depends on the amplitude of the optically induced field pulse: if the pulse amplitude $H_{\mathrm{P}}<H_{\mathrm{C}}$, the system relaxes back to the initial state $\varphi=0$; for $H_{\mathrm{P}}>H_{\mathrm{C}}$, the resulting state is the $\Gamma_{24}$ phase with $\varphi= \pm \pi / 2$. The value of the critical field for $\mathrm{HoFeO}_{3}$ is of the order of $0.5 \mathrm{~T}$. Figure 3 shows that the coordinate $\varphi$ of the spin hardly changes during the $100 \mathrm{fs}$ action of the magnetic field. Instead, the spins acquire sufficient momentum to overcome the potential barrier afterwards. The short magnetic pulse triggers a motion with an essentially constant value $\mathrm{d} \varphi / \mathrm{d} t$. The motion persists long after the pulse is gone. The speed of overcoming the potential barrier is given by the frequency of the spin-wave resonance in the magnet and for this particular case is equal to $0.5 \mathrm{rad} \mathrm{ps}^{-1}$. This is much faster than the speed of spin switching measured earlier for ferromagnets $\left(\sim 0.0314 \mathrm{rad} \mathrm{ps}^{-1}\right.$; refs $\left.4-7\right)$.

To observe this inertial motion of spins in $\mathrm{HoFeO}_{3}$ we carried out pump-probe experiments with $100 \mathrm{fs}$ laser pulses, producing an effective magnetic field by a circularly polarized pump pulse owing to the inverse Faraday effect. The field is proportional to $\left[\mathbf{E}(\omega) \times \mathbf{E}^{*}(\omega)\right]$, where $\mathbf{E}(\omega)$ is the electric field of the light wave at frequency $\omega$, and present in the medium only during the action of the laser pulse ${ }^{14,15}$. Rare-earth orthoferrites are known to possess large inverse Faraday effects resulting in effective magnetic fields up to $1 \mathrm{~T}$ (ref. 16), directed along the wavevector of light. Right- and left-handed circularly polarized pulses act as fields of opposite orientation. The pump-induced spin dynamics was monitored by detecting the $M_{Z}$ component of the magnetization through the magneto-optical Faraday effect in the probe pulse, measured as a function of delay between the pump and probe pulses. The results of such time-resolved measurements are shown in Fig. $3 \mathrm{~b}$ for the two opposite pump helicities. The experimental observations are in excellent agreement with the 
results of the simulations and are an experimental demonstration of an inertial motion of antiferromagnetically coupled spins in $\mathrm{HoFeO}_{3}$, triggered by a short optically generated magnetic-field pulse. Outside the range between $T_{1}=38 \mathrm{~K}$ and $T_{\mathrm{t}}=52 \mathrm{~K}$, such pulses cause only small-amplitude damped oscillations towards the initial state, similar to that observed in ref. 16. This again strongly confirms the original hypothesis that the dynamics shown in Fig. 3 is related to a reorientation from the $\Gamma_{12}$ into the $\Gamma_{24}$ magnetic phase.

It is interesting to note that although the two magnetic phases coexist between $T_{1}=38 \mathrm{~K}$ and $T_{2}=58 \mathrm{~K}$, in the stroboscopic pump-probe experiment the switching from the $\Gamma_{12}$ to the $\Gamma_{24}$ phase can be seen only in the range from $T_{1}=38 \mathrm{~K}$ and $T_{\mathrm{t}}=52 \mathrm{~K}$. Below $52 \mathrm{~K}$, the $\Gamma_{12}$ phase has lower energy and the switching of the spin system from $\Gamma_{12}$ to $\Gamma_{24}$ will be followed by thermal relaxation so that the system will be thermodynamically restored into the initial $\Gamma_{12}$ state. Above $52 \mathrm{~K}, \Gamma_{24}$ has a lower energy, and after the spins are switched from $\Gamma_{12}$ to $\Gamma_{24}$, their initial state is not restored and the switching process cannot be observed in a stroboscopic experiment.

It must be mentioned that the agreement between theory and experiment is observed only within the first few hundred picoseconds after the laser pulse action (see Fig. 4a). Time-resolved measurements of the Faraday rotation at different temperatures and for time delays up to 1.2 ns clearly show that the difference between experiments with opposite pump helicities vanishes beyond $600 \mathrm{ps}$. To explain such laser-induced magnetic changes for longer delays we should note that owing to absorption $\left(k=300 \mathrm{~cm}^{-1}\right)$ the excitation of the material with the laser pulse leads to a rapid increase of lattice temperature of about $12 \mathrm{~K}$. Such a heating can also result in a spin-reorientation between the phases $\Gamma_{12}$ and $\Gamma_{24}$. However, in contrast to the effect triggered by a laser-induced effective magnetic field as discussed above, such a reorientation does not depend on the helicity of the optical excitation. To separate the field-driven and heat-driven spin reorientation we should separate the helicity-dependent and helicity-independent contributions to the magneto-optical rotation, respectively. These contributions were calculated as the difference and the sum of the Faraday rotation measured for the two opposite pump helicities. These quantities were normalized on the Faraday rotation at $T_{\mathrm{t}}=52 \mathrm{~K}\left(0.27^{\circ}\right)$ and the results are shown in Fig. $4 \mathrm{~b}$ in terms of laser-induced changes of the magnetization $M_{Z}$. The figure clearly shows that the spin reorientation triggered by a laser-generated effective magnetic field is two orders of magnitude faster than the similar reorientation triggered by a rapid temperature increase. Moreover, the time constant (of about $200 \mathrm{ps}$ ) of the relaxation of the helicity-dependent contribution to $\Delta M_{Z}$ remarkably coincides with the time constant of the heat-driven increase of $\Delta M_{Z}$ (see Fig. 4b). Such a slow dynamics can be explained as a relaxation of a medium towards thermal equilibrium through the relaxation of unfavourable and growth of favourable $\Gamma_{24}$ domains. Note that the temperature dependencies of the efficiency of both heat-induced and field-induced magnetic changes are very typical for non-uniform laser-induced phase transitions $^{17,18}$ (see Fig. 4c). The fact that $\Delta M_{Z /} / M_{Z}$ averaged over the probed volume was only about $8 \%$ is most probably caused by a non-uniformity of pump intensity over the probed volume. It is important to stress that the absolute value of the Faraday effect in orthoferrites is seriously influenced by linear birefringence. In particular, it has been shown that because of the linear birefringence the amplitude of the laser-induced magnetic changes probed through the Faraday effect in orthoferrites can be easily underestimated ${ }^{19,20}$.

The new mechanism of spin switching based on the inertia of the spin system in antiferromagnets, as demonstrated here, enables us to trigger the writing of one bit of information using extremely short magnetic-field pulses. Such an inertial mechanism of magnetic writing opens new opportunities for sequential writing of a large number of data, for example the realization of threedimensional displays and many other applications where reaching the ultimate speed of addressing one bit is extremely important, whereas the actual switching of one bit between two states may occur at a much slower timescale. Finally, we would like to note that inertia-driven spin switching is not limited to antiferromagnets. Even if a subpicosecond magnetic pulse alone cannot switch the magnetization of a single ferromagnetic layer ${ }^{3}$ and the assistance of a strong electric field is required ${ }^{21}$, in a heterostructure made of two ferromagnetic layers with a strong antiferromagnetic coupling (as in a so-called artificial antiferromagnet used for exchange biasing GMR-type sensors), switching with an ultrashort magnetic pulse is possible through the inertial mechanism.

\section{Methods}

Experiment. To monitor the magnetization dynamics in $\mathrm{HoFeO}_{3}$, the Faraday effect, that is the rotation of the polarization plane of light with wavevector $\mathbf{k}$ transmitted through the sample with magnetization $\mathbf{M}$, was measured, $\alpha_{\mathrm{F}}=\chi \mathbf{M k}$ ( $\chi$ is the magneto-optical susceptibility $)^{8}$. In the experiments we used thick $60-\mu \mathrm{m}$ plates of $\mathrm{HoFeO}_{3}$ cut perpendicularly to the $z$ axis. The time-resolved measurements of the Faraday rotation were made in a pump-and-probe configuration at a photon energy of $1.52 \mathrm{eV}$ using amplified 100-fs pulses from a Ti:sapphire laser at a repetition rate of $1 \mathrm{kHz}$. The intensity ratio between the pump and probe pulses was about 100. Both beams were focused on the sample to a spot diameter of about $100 \mu \mathrm{m}$ for the pump and somewhat smaller for the probe beam. The pump fluence on the sample was around $10 \mathrm{~mJ} \mathrm{~cm}{ }^{-2}$. The experiments were carried out at normal incidence and in a magnetic field of about $H_{\text {appl }}=100$ Oe applied along the $z$ axis. To separate the transient magneto-optical Faraday effect from possible artefacts, we have measured the polarization rotation $\alpha$ or the two polarities of the field and have taken the difference $\alpha_{\mathrm{F}}=\alpha\left(+H_{\text {appl }}\right)-\alpha\left(-H_{\text {appl }}\right)$.

Theory. In equation (3), the anisotropy energy is modelled by standard expansion over components of the vector $\mathbf{l}$ to fourth order. For a magnetic field pulse of short duration $\Delta t \ll 1 / \omega_{0}$, equation (3) was solved numerically and analytically, replacing the real pulse shape by a Dirac delta function, $H(t) \rightarrow H_{\mathrm{p}} \Delta t \delta(t)$, $H_{\mathrm{p}} \Delta=\int H(t) \mathrm{d} t$. Then before $(t<0)$ and after $(t>0)$ the action of the pulse, the spin dynamics is described by equation (3) with $H(t)=0$. Assuming $\varphi=0$ at $t<0$ it is easy to find the initial conditions of the form $\varphi=0, d \varphi / \mathrm{d} t=\gamma^{2} H_{\mathrm{D}} H_{\mathrm{p}} \Delta t / \sin \theta_{0}$, which gives the solution of equation (3) in the same form of a damped nonlinear oscillation as in Fig. 3.

Received 11 June 2008; accepted 16 July 2009; published online 23 August 2009

\section{References}

1. Stöhr, J. \& Siegmann, H. C. Magnetism: From Fundamentals to Nanoscale Dynamics (Springer, 2006).

2. Back, C. H. et al. Minimum field strength in precessional magnetization reversal. Science 285, 864-867 (1999).

3. Tudosa, I. et al. The ultimate speed of magnetic switching in granular recording media. Nature 428, 831-833 (2004).

4. Kaka, S. \& Russek, S. E. Precessional switching of submicrometer spin valves. Appl. Phys. Lett. 80, 2958-2960 (2002).

5. Schumacher, H. W., Chappert, C., Sousa, R. C., Freitas, P. P. \& Miltat, J. Quasiballistic magnetization reversal. Phys. Rev. Lett. 90, 017204 (2003).

6. Gerrits, Th., van den Berg, H. A. M., Hohlfeld, J., Bär, L. \& Rasing, Th. Ultrafast precessional magnetization reversal by picosecond magnetic field pulse shaping. Nature 418, 509-512 (2002).

7. Hiebert, W. K., Lagae, L. \& De Boeck, J. Spatially inhomogeneous ultrafast precessional magnetization reversal. Phys. Rev. B 68, 020402 (2003).

8. Landau, L. D. \& Lifshitz, E. M. Electrodynamics of Continuous Media (Pergamon, 1984).

9. Baryakhtar, V. G., Ivanov, B. A. \& Chetkin, M. V. Dynamics of domain boundaries in weak ferromagnets. Sov. Phys. Usp 28, 563-588 (1985).

10. Baryakhtar, V. G., Ivanov, B. A., Chetkin, M. V. \& Gadetskii, S. N. Dynamics of Topological Magnetic Solitons. Experiment and Theory (Springer Tract in Modern Physics 139, Springer, 1994).

11. Vorob'ev, G. P., Kadomtseva, A. M., Krynetskii, I. B. \& Mukhin, A. A. Unusual nature of spin reorientation in $\mathrm{HoFeO}_{3}$. Sov. Phys. JETP 68, 604-628 (1989).

12. Balbashov, A. M. et al. Anomalies of high-frequency magnetic-properties and new reorientation transitions in $\mathrm{HoFeO}_{3}$. Sov. Phys. JETP 68, 629-638 (1989).

13. Wijn, H. P. J. (ed.) Landolt-Börnstein New Series Group III Vol. 27, f3, 134-138 (Springer, 1981). 
14. Pitaevskii, L. P. Electric forces in a transparent dispersive medium. Sov. Phys. JETP 12, 1008-1013 (1961).

15. van der Ziel, J. P., Pershan, P. S. \& Malmstrom, L. D. Optically-induced magnetization resulting from the inverse Faraday effect. Phys. Rev. Lett. 15, 190-193 (1965).

16. Kimel, A. V. et al. Ultrafast nonthermal control of magnetization by instantaneous photomagnetic pulses. Nature 435, 655-657 (2005).

17. Kimel, A. V., Kirilyuk, A., Tsvetkov, A., Pisarev, R. V. \& Rasing, Th. Laser-induced ultrafast spin reorientation in the antiferromagnet $\mathrm{TmFeO}_{3}$. Nature 429, 850-853 (2004).

18. Kimel, A. V., Pisarev, R. V., Hohlfeld, J. \& Rasing, Th. Ultrafast quenching of the antiferromagnetic order in $\mathrm{FeBO}_{3}$ : Direct optical probing of the phonon-magnon coupling. Phys. Rev. Lett. 89, 287401 (2002).

19. Tabor, W. J. \& Chen, F. S. Propagation through materials possessing both Faraday rotation and birefringence: Experiments with ytterbium orthoferrite J. Appl. Phys. 40, 2760-2765 (1969).

20. Woodford, S. R., Bringer, A. \& Blügel, S. Interpreting magnetization from Faraday rotation in birefringent magnetic media. J. Appl. Phys. 101, 053912 (2007).

21. Gamble, S. J. et al. Electric field induced magnetic anisotropy in a ferromagnet. Phys. Rev. Lett. 102, 217201 (2009).

\section{Acknowledgements}

The authors thank A. M. Balbashov for providing the samples and E. Galkina for her help with calculations as well as C. D. Stanciu, T. Toonen and A. van Etteger for their help with the measurements. This work was partially supported by INTAS (project 1000008-8112), the Russian Foundation for Basic Research (RFBR), joint grant 219-09 of the Ukrainian Academy of Science and RFBR, de Nederlandse Organisatie voor Wetenschappelijk Onderzoek (NWO) and de Stichting voor Fundamenteel Onderzoek der Materie (FOM) as well as the Dutch nanotechnology initiative NanoNed and the European Community's Seventh Framework Programme (FP7/2007-2013) under grant agreements NMP3-SL-2008-214469 (UltraMagnetron) and N 214810 (FANTOMAS).

\section{Author contributions}

A.V.K., R.V.P., P.A.U., A.K. and Th.R. initiated the project, being responsible for the experimental work. B.A.I. is responsible for the theoretical part.

\section{Additional information}

Supplementary information accompanies this paper on www.nature.com/naturephysics. Reprints and permissions information is available online at http://npg.nature.com/ reprintsandpermissions. Correspondence and requests for materials should be addressed to A.V.K. 